\title{
The Split Common Fixed Point Problem for $\varrho$-Strictly Pseudononspreading Mappings
}

\author{
Shubo Cao ${ }^{1,2}$ \\ ${ }^{1}$ School of Management, Tianjin University, Tianjin 300072, China \\ ${ }^{2}$ School of Economics and Management, Civil Aviation University of China, Tianjin 300300, China
}

Correspondence should be addressed to Shubo Cao; tjsbcao@163.com

Received 10 January 2013; Revised 12 March 2013; Accepted 11 April 2013

Academic Editor: Filomena Cianciaruso

Copyright (c) 2013 Shubo Cao. This is an open access article distributed under the Creative Commons Attribution License, which permits unrestricted use, distribution, and reproduction in any medium, provided the original work is properly cited.

We introduce and analyze the viscosity approximation algorithm for solving the split common fixed point problem for the strictly pseudononspreading mappings in Hilbert spaces. Our results improve and develop previously discussed feasibility problems and related results.

\section{Introduction}

Throughout this paper, we always assume that $H$ is a real Hilbert space with inner product $\langle\cdot, \cdot\rangle$ and norm $\|\cdot\|$. Let $I$ denote the identity operator on $H$. Let $H_{1}$ and $H_{2}$ be two real Hilbert spaces and let $A: H_{1} \rightarrow H_{2}$ be a bounded linear operator. Given closed convex subsets $C$ and $Q$ of $H_{1}$ and $H_{2}$, respectively.

The split feasibility problem (SFP) (Censor and Elfving 1994 [1]), modeling phase retrieval problems, is to find a point $x^{*}$ with the property

$$
x^{*} \in C, \quad A x^{*} \in D .
$$

Recently, it has been found that the SFP can also be used to model the intensity-modulated radiation therapy [2-8]. A special case of the SFP (1) is the convexly constrained linear problem:

$$
A x=b, \quad x \in C .
$$

This problem, due to its applications in many applied disciplines, has extensively been investigated in the literature ever since Landweber [9] introduced his iterative method in 1951.

Note that the split feasibility problem (1) can be formulated as fixed point equation by using the fact

$$
x^{*}=P_{\mathrm{C}}\left(I-\gamma A^{*}\left(I-P_{\mathrm{Q}}\right) A\right) x^{*},
$$

where $P_{C}$ and $P_{\mathrm{Q}}$ are the projections onto $C$ and $Q$, respectively, $\gamma>0$ is any positive constant, and $A^{*}$ denotes the adjoint of $A$; that is, $x^{*}$ solves the SFP (1) if and only if $x^{*}$ solves the fixed point equation (3) (see [10] for more details). This implies that we can use fixed point algorithms to solve SFP.

In 2002, Byrne [2] proposed his CQ algorithm to solve (1). The sequence $\left\{x_{n}\right\}$ is generated by the following iteration scheme:

$$
x_{n+1}=P_{C}\left(I-\gamma A^{*}\left(I-P_{\mathrm{Q}}\right) A\right) x_{n}, \quad n \in N,
$$

where $\gamma \in(0,2 / \lambda)$, with $\lambda$ being the spectral radius of the operator $A^{*} A$.

The CQ algorithm (4) is a special case of the K-M algorithm. Due to the fixed point formulation (2) of the SFP, Moudafi [11] applied the K-M algorithm to the operator $P_{C}\left(I-\gamma A^{*}\left(I-P_{\mathrm{Q}}\right) A\right)$ to obtain a sequence given by

$$
\begin{array}{r}
x_{n+1}=\left(1-\alpha_{n}\right) x_{n}-\alpha_{n} P_{C}\left(I-\gamma A^{*}\left(I-P_{\mathrm{Q}}\right) A\right) x_{n}, \\
n \in N,
\end{array}
$$

where $\gamma \in(0,2 / \lambda)$, with $\lambda$ being the spectral radius of the operator $A^{*} A$, and the sequence $\left\{\alpha_{n}\right\}$ satisfies the condition $\sum_{n=1}^{\infty} \alpha_{n}\left(1-\alpha_{n}\right)=+\infty$; he proved weak convergence result of the algorithm (5) in Hilbert spaces.

In 2009, Censor and Segal [12] considered the following algorithm to be solved (1). 
Algorithm 1. Initialization: let $x^{*} \in H_{1}=R^{n}$ be arbitrary. Iterative step: for $n \in N$ let

$$
x_{n+1}=U\left(x_{n}+\gamma A^{*}\left((V-I) A x_{n}\right)\right), \quad n \in N \text {, }
$$

where $\gamma \in(0,2 / \lambda)$ with $\lambda$ being the spectral radius of the operator $A^{*} A$ and $U, V$ be a single pair of directed operators.

In 2010, Moudafi [13] extended the Algorithm 1 and introduced the following algorithm with weak convergence for the split common fixed point problem.

Algorithm 2. Initialization: let $x^{*} \in H_{1}=R^{n}$ be arbitrary.

Iterative step: for $n \in N$ let

$$
\begin{gathered}
u_{n}=x_{n}+\gamma \beta A^{*}(V-I) A x_{n}, \\
x_{n+1}=\left(1-\alpha_{n}\right) u_{n}+\alpha_{n} U\left(u_{n}\right), \quad n \in N,
\end{gathered}
$$

where $\beta \in(0,1), \alpha_{n} \in(0,1)$, and $\gamma \in(0,1 / \lambda \beta)$ with $\lambda$ being the spectral radius of the operator $A^{*} A$ and $U, V$ be a pair of quasi-nonexpansive operators.

In 2012, Zhao and He [14] continue to consider the split common fixed point problem with quasi-nonexpansive operators and to use the following algorithm to obtain the strong convergence of the viscosity method for solving the split common fixed point problem.

Algorithm 3. Initialization: let $x^{*} \in H_{1}=R^{n}$ be arbitrary. Iterative step: for $n \in N$ let

$$
\begin{aligned}
& \quad T=U\left(I+\gamma A^{*}(V-I) A\right) \\
& x_{n+1}=\alpha_{n} f\left(x_{n}\right)+\left(1-\alpha_{n}\right) \\
& \times\left(\left(1-\omega_{n}\right) x_{n}+\omega_{n} T x_{n}\right), \quad n \in N,
\end{aligned}
$$

where $f: H \rightarrow H$ is a contractive mapping with constant $\beta \in(0,1), \alpha_{n} \in(0,1)$, and $\gamma \in(0,1 / \lambda)$ with $\lambda$ being the spectral radius of the operator $A^{*} A$ and $U, V$ be a pair of quasinonexpansive operators.

Motivated and inspired by Censor and Segal [12], Moudafi [11], and Zhao and $\mathrm{He}$ [14], we introduce the following relaxed algorithm.

Algorithm 4. Initialization: let $x^{*} \in H_{1}=R^{n}$ be arbitrary. Iterative step: for $n \in N$ let

$$
\begin{gathered}
T=U\left(I+\gamma A^{*}(V-I) A\right) \\
x_{n+1}=\alpha_{n} \sigma f\left(x_{n}\right)+\left(I-\mu \alpha_{n} B\right) \\
\times\left(\left(1-\omega_{n}\right) x_{n}+\omega_{n} T x_{n}\right), \quad n \in N,
\end{gathered}
$$

where $f: H \rightarrow H$ is a contractive mapping with constant $\beta \in(0,1), B: H \rightarrow H$ is $\eta$-strongly monotone and boundedly Lipschitzian, $\alpha_{n} \in(0,1)$, and $\gamma \in(0,1 / \lambda)$ with $\lambda$ being the spectral radius of the operator $A^{*} A$ and $U, V$ be a pair of $\varrho_{i}$-strictly pseudononspreading mappings $i=1,2$.
This paper establishes the strong convergence of the sequence given by (9) to the unique solution of solving the split common fixed point problem and the following variational inequality problem $\operatorname{VIP}(\mu B-\sigma f, T)$ :

$$
\text { find } \begin{aligned}
x^{*} \in \Gamma \text { such that }\left\langle(\mu B-\sigma f) x^{*}, v-x^{*}\right\rangle & \geq 0, \\
v & \in \Gamma .
\end{aligned}
$$

\section{Preliminaries}

In this section, we introduce the concepts of contraction mappings, nonexpansive mappings, quasi-nonexpansive mappings, and $\varrho$-strictly pseudononspreading mappings and some Lemmas.

Assume that $C$ is a nonempty closed and convex subset of Hilbert space $H$. Recall that the (nearest point or metric) projection from $H$ onto $K$, that denoted $P_{C}$, assigns, to each $x \in H$, the unique point $P_{C} x \in C$ with the property

$$
\left\|x-P_{C} x\right\|=\inf \{\|x-y\|: y \in C\} .
$$

Definition 5. A mapping $T: H \rightarrow H$ is said to be

(1) contraction, if $\|T x-T y\| \leq \beta\|x-y\|, \forall x, y \in H$ and $\beta \in(0,1)$;

(2) nonexpansive, if $\|T x-T y\| \leq\|x-y\|, \forall x, y \in H$;

(3) quasi-nonexpansive, $\|T x-q\| \leq\|x-q\|, \forall(x, q) \in$ $H \times F_{i x}(T)$.

Remark 6. From the Definition 5, It is easy to see that

(i) iterative methods for quasi-nonexpansive mappings have been extensively investigated; see [13-17];

(ii) a nonexpansive mapping is a quasi-nonexpansive mapping.

Following the terminology of Browder and Petryshyn [18], we obtain the following definitions.

Definition 7. A mapping $T: D(T) \subseteq H \rightarrow H$ is $\varrho$-strictly pseudononspreading if there exists $\varrho \in[0,1)$ such that

$$
\begin{gathered}
\|T x-T y\|^{2} \leq \\
+x-y\left\|^{2}+\varrho\right\| x-T x-(y-T y) \|^{2} \\
+2\langle x-T x, y-T y\rangle
\end{gathered}
$$

for all $x, y \in D(T)$.

Iterative methods for strictly pseudononspreading mapping have been extensively investigated; see [19-23].

Lemma 8 (see [24]). Let $H$ be a Hilbert spaces, and $f: H \rightarrow$ $H$ is a contractive mapping with constant $\beta \in(0,1) . B: H \rightarrow$ $H$ is $k$-Lipschitzian and $\eta$-strongly monotone operator with $k>$ $0, \eta>0$. Then for $0<\sigma<\mu \eta / \beta$,

$$
\begin{aligned}
& \langle x-y,(\mu B-\sigma f) x-(\mu B-\sigma f) y\rangle \\
& \geq(\mu \eta-\sigma \beta)\|x-y\|^{2}, \quad x, y \in H .
\end{aligned}
$$

That is, $\mu B-\sigma f$ is strongly monotone with coefficient $\mu \eta-\sigma \beta$. 
Lemma 9. Let $H$ be a real Hilbert space. Then the following well-known results hold: for all $x, y \in H$ and $t \in[0,1]$

(i) $\|x+y\|^{2} \leq\|x\|^{2}+2\langle y, x+y\rangle$;

(ii) $\|t x+(1-t) y\|^{2}=t\|x\|^{2}+(1-t)\|y\|^{2}-t(1-t)\|x-y\|^{2}$;

(iii) $\langle x, y\rangle=-(1 / 2)\|x-y\|^{2}+(1 / 2)\|x\|^{2}+(1 / 2)\|y\|^{2}$.

Lemma 10. Let $T$ be a $\varrho$-strictly pseudononspreading mapping with $\varrho \in(0,1)$, and set $T_{\alpha}=(1-\alpha) I+\alpha T, \alpha \in(\varrho, 1)$. The following properties are reached for each $(x, p) \in H \times F_{i x}(T)$ :

(1) $\langle x-T x, x-p\rangle \geq((1-\varrho) / 2)\|x-T x\|^{2}$ and $\langle x-T x, p-$ $T x\rangle \leq((1+\varrho) / 2)\|x-T x\|^{2} ;$

(2) $\left\|T_{\alpha} x-p\right\|^{2} \leq\|x-p\|^{2}-\alpha(1-\varrho-\alpha)\|x-T x\|^{2}$;

(3) $\left\langle x-T_{\alpha} x, x-p\right\rangle \geq(\alpha(1-\varrho) / 2)\|x-T x\|^{2}$.

Proof. Note that property (1) is easily deduced from the Lemma 8 (iii) and the fact that $T$ is $\varrho$-strictly pseudononspreading mapping, we obtain

$$
\begin{aligned}
& \langle x-T x, x-p\rangle=-\frac{1}{2}\|T x-p\|^{2}+\frac{1}{2}\|x-T x\|^{2} \\
& +\frac{1}{2}\|x-p\|^{2} \\
& =-\frac{1}{2}\|T x-p\|^{2}+\frac{1-\varrho}{2}\|x-T x\|^{2} \\
& +\frac{1}{2}\left(\|x-p\|^{2}+\varrho\|x-T x\|^{2}\right) \\
& \geq-\frac{1}{2}\|T x-p\|^{2}+\frac{1}{2}\|T x-p\|^{2} \\
& +\frac{1-\varrho}{2}\|x-T x\|^{2} \\
& \geq \frac{1-\varrho}{2}\|x-T x\|^{2}, \\
& \langle x-T x, p-T x\rangle=-\frac{1}{2}\|x-p\|^{2}+\frac{1}{2}\|x-T x\|^{2} \\
& +\frac{1}{2}\|T x-p\|^{2} \\
& \leq-\frac{1}{2}\|T x-p\|^{2}+\|T x-p\|^{2} \\
& +\left(\frac{1}{2}+\varrho\right)\|T x-p\|^{2} \\
& \leq \frac{1+\varrho}{2}\|x-T x\|^{2} \text {. }
\end{aligned}
$$

Property (2) is obtained from property (1) and by

$$
\begin{aligned}
\left\|T_{\alpha} x-p\right\|^{2}= & \|x-p\|^{2}-2 \alpha\langle x-p, x-T x\rangle \\
& +\alpha^{2}\|T x-x\|^{2} .
\end{aligned}
$$
(1).

Property (3) is given by $I-T_{\alpha}=\alpha(I-T)$ and property
Lemma 11 (see [25]). Let $\left\{\mathscr{T}_{n}\right\}$ be a sequence of real numbers that does not decrease at infinity, in the sense that there exists a subsequence $\left\{\mathscr{T}_{n_{j}}\right\}_{j \geq 0}$ of $\left\{\mathscr{T}_{n}\right\}$ which satisfies $\mathscr{T}_{n_{j}}<\mathscr{T}_{n_{j}+1}$ for all $j \geq 0$. Also consider the sequence of integers $\{\delta(n)\}_{n \geq n_{0}}$ defined by

$$
\delta(n)=\max \left\{k \leq n \mid \mathscr{T}_{k}<\mathscr{T}_{k+1}\right\}
$$

Then $\{\delta(n)\}_{n \geq n_{0}}$ is a nondecreasing sequence verifying $\lim _{n \rightarrow \infty} \delta(n)=\infty$, for all $n \geq n_{0}$; it holds that $\mathscr{T}_{\delta(n)}<\mathscr{T}_{\delta(n)+1}$ and one has

$$
\mathscr{T}_{n}<\mathscr{T}_{\delta(n)+1}
$$

Lemma 12. Let $K$ be a closed convex subset of a real Hilbert space $H$, given $x \in H$ and $y \in K$. Then $y=P_{K} x$ if and only if there holds the inequality

$$
\langle x-y, y-z\rangle \geq 0, \quad \forall z \in K \text {. }
$$

\section{Main Results}

In what follows, we will focus our attention on the following general two operator split common fixed point problem in real Hilbert space $H$ :

$$
\text { find } x^{*} \in C \text { such that } A x^{*} \in D \text {, }
$$

where $A: H_{1} \rightarrow H_{2}$ is a bounded linear operator, $U: H_{1} \rightarrow$ $H_{1}$ and $V: H_{2} \rightarrow H_{2}$ are two $\varrho_{i}$-strictly pseudononspreading mappings $i=1,2$ with nonempty fixed point sets $F_{i x}(U)=$ $C$ and $F_{i x}(V)=Q$, and denote the solution set of the twooperator SCFP by

$$
\Gamma=\{y \in C ; A y \in Q\}
$$

On the other hand, $x^{*} \in \Gamma$ is also unique solution of solving the variational inequality problem $\operatorname{VIP}(\mu B-\sigma f, T)$ :

$$
x^{*} \in \Gamma, \quad\left\langle(\mu B-\sigma f) x^{*}, y-x^{*}\right\rangle \geq 0, \quad y \in \Gamma,
$$

where $B: H \rightarrow H$ is $\eta$-strongly monotone and $k$-Lipschitzian on $H$ with $k>0, \eta>0$. Let $0<\mu<2 \eta / k^{2}, 0<\sigma<$ $\mu\left(\eta-\left(\mu k^{2} / 2\right)\right) / \beta=\tau / \beta$.

Before stating our main convergence result, we establish the boundedness of the iterates given by (9).

Lemma 13. The sequence $\left\{x_{n}\right\}$ is generated by (9), and let $U: H_{1} \rightarrow H_{1}$ and $V: H_{2} \rightarrow H_{2}$ be two $\varrho_{i}$-strictly pseudononspreading mappings on $H, i=1,2$, and $f: H \rightarrow H$ is a contractive mapping with constant $\beta \in(0,1),\left\{\alpha_{n}\right\} \subset(0,1)$ and $0<\varrho_{i}<\omega_{n}<1 / 2, i=1,2$. Then $\left\{x_{n}\right\}$ is bounded.

Proof. Set $T_{\omega_{n}}=\left(1-\omega_{n}\right) I+\omega_{n} T$. Then $x_{n+1}=\alpha_{n} \sigma f\left(x_{n}\right)+(I-$ $\left.\mu \alpha_{n} B\right) T_{\omega_{n}} x_{n}$ 

obtain

Taking $y \in \Gamma$, that is, $y \in F_{i x}(U)$ and $A y \in F_{i x}(V)$. We

$$
\begin{aligned}
\left\|x_{n+1}-y\right\|= & \| \alpha_{n} \sigma\left(f\left(x_{n}\right)-f(y)\right)+\alpha_{n}(\sigma f(y)-\mu B y) \\
& +\left(I-\mu \alpha_{n} B\right)\left(T_{\omega_{n}} x_{n}-y\right) \| \\
\leq & \alpha_{n} \sigma\left\|f\left(x_{n}\right)-f(y)\right\|+\alpha_{n}\|\sigma f(y)-\mu B y\| \\
& +\left(1-\alpha_{n} \tau\right)\left\|T_{\omega_{n}} x_{n}-y\right\| \\
\leq & \alpha_{n} \sigma \beta\left\|x_{n}-y\right\|+\alpha_{n}\|\sigma f(y)-\mu B y\| \\
& +\left(1-\alpha_{n} \tau\right)\left\|T_{\omega_{n}} x_{n}-y\right\| .
\end{aligned}
$$

From the definition of $T_{\omega_{n}}$, we have

$$
\begin{aligned}
\left\|T_{\omega_{n}} x_{n}-y\right\|^{2}= & \left\|\left(1-\omega_{n}\right) x_{n}+\omega_{n} T x_{n}-y\right\|^{2} \\
= & \left\|x_{n}-y+\omega_{n}\left(T x_{n}-x_{n}\right)\right\|^{2} \\
= & \left\|x_{n}-y\right\|^{2}-2 \omega_{n}\left\langle x_{n}-y, x_{n}-T x_{n}\right\rangle \\
& +\omega_{n}^{2}\left\|T x_{n}-x_{n}\right\|^{2} .
\end{aligned}
$$

On the other hand, we obtain

$$
\begin{aligned}
\left\|T x_{n}-y\right\|^{2}= & \left\|U\left(I+\gamma A^{*}(V-I) A\right) x_{n}-y\right\|^{2} \\
\leq & \left\|\left(I+\gamma A^{*}(V-I) A\right) x_{n}-y\right\|^{2} \\
= & \left\|x_{n}-y\right\|^{2}+\gamma^{2}\left\|A^{*}(V-I) A x_{n}\right\|^{2} \\
& +2 \gamma\left\langle x_{n}-y, A^{*}(V-I) A x_{n}-y\right\rangle \\
= & \left\|x_{n}-y\right\|^{2}+\gamma^{2}\left\langle(V-I) A x_{n}, A A^{*}(V-I) A x_{n}\right\rangle \\
& +2 \gamma\left\langle x_{n}-y, A^{*}(V-I) A x_{n}\right\rangle .
\end{aligned}
$$

According to the definition of $\lambda$, we have

$$
\begin{aligned}
& \gamma^{2}\left\langle(V-I) A x_{n}, A A^{*}(V-I) A x_{n}\right\rangle \\
& \quad \leq \lambda \gamma^{2}\left\langle(V-I) A x_{n},(V-I) A x_{n}\right\rangle \\
& \quad=\lambda \gamma^{2}\left\|(V-I) A x_{n}\right\|^{2} .
\end{aligned}
$$

Now, by using property (1) of Lemma 9 , we obtain

$$
\begin{aligned}
2 \gamma\left\langle x_{n}-y, A^{*}(V-I) A x_{n}\right\rangle & \\
= & 2 \gamma\left\langle A\left(x_{n}-y\right),(V-I) A x_{n}\right\rangle \\
= & 2 \gamma\left\langle A\left(x_{n}-y\right)+(V-I) A x_{n}\right. \\
& \left.-(V-I) A x_{n},(V-I) A x_{n}\right\rangle
\end{aligned}
$$$$
=2 \gamma\left(\left\langle V A x_{n}-A y,(V-I) A x_{n}\right\rangle\right.
$$$$
\left.-\left\|(V-I) A x_{n}\right\|^{2}\right)
$$$$
\leq 2 \gamma\left(\frac{1-\varrho_{2}}{2}\left\|(V-I) A x_{n}\right\|^{2}\right.
$$$$
\left.-\left\|(V-I) A x_{n}\right\|^{2}\right)
$$$$
\leq-\gamma\left(1-\varrho_{2}\right)\left\|(V-I) A x_{n}\right\|^{2} .
$$

Combining (24)-(26), we obtain

$$
\begin{aligned}
\left\|T x_{n}-y\right\|^{2}= & \left\|x_{n}-y\right\|^{2}+\lambda \gamma^{2}\left\|(V-I) A x_{n}\right\|^{2} \\
& -\gamma\left(1-\varrho_{2}\right)\left\|(V-I) A x_{n}\right\|^{2} \\
= & \left\|x_{n}-y\right\|^{2}-\gamma\left(\left(1-\varrho_{2}\right)-\lambda \gamma\right) \\
& \times\left\|(V-I) A x_{n}\right\|^{2} \\
\leq & \left\|x_{n}-y\right\|^{2} .
\end{aligned}
$$

From property (i) of Lemma 9 and (23), we get

$$
\begin{aligned}
\left\|T_{\omega_{n}} x_{n}-y\right\|^{2} \leq & \left\|x_{n}-y\right\|^{2}-\left(1-\varrho_{2}\right) \omega_{n}\left\|x_{n}-T x_{n}\right\|^{2} \\
& +\omega_{n}^{2}\left\|T x_{n}-x_{n}\right\|^{2} \\
= & \left\|x_{n}-y\right\|^{2}-\omega_{n}\left(1-\varrho_{2}-\omega_{n}\right) \\
& \times\left\|x_{n}-T x_{n}\right\|^{2} \\
\leq & \left\|x_{n}-y\right\|^{2} .
\end{aligned}
$$

Combining (22), (23), and (28), we have

$$
\begin{aligned}
\left\|x_{n+1}-y\right\| \leq & \alpha_{n} \sigma \beta\left\|x_{n}-y\right\|+\alpha_{n}\|f(y)-\mu B y\| \\
+ & \left(1-\alpha_{n} \tau\right)\left\|T_{\omega_{n}} x_{n}-y\right\| \\
= & {\left[1-\alpha_{n}(\tau-\sigma \beta)\right]\left\|x_{n}-y\right\| } \\
& +\alpha_{n}\|f(y)-\mu B y\| \\
\leq & \max \left\{\left\|x_{n}-y\right\|, \frac{1}{\tau-\sigma \beta}\|f(y)-\mu B y\|\right\} .
\end{aligned}
$$

It follows from (29) and induction that

$$
\left\|x_{n+1}-y\right\| \leq \max \left\{\left\|x_{0}-y\right\|, \frac{1}{\tau-\sigma \beta}\|f(y)-\mu B y\|\right\},
$$

and hence $\left\{x_{n}\right\}$ is bounded.

Now we are in position to claim the main convergence result. 
Theorem 14. Given a bounded linear operator $A: H_{1} \rightarrow H_{2}$, let $U: H_{1} \rightarrow H_{1}$ and $V: H_{2} \rightarrow H_{2}$ be two $\varrho_{i}$-strictly pseudononspreading mappings, and $i=1,2$ with fixed point $F_{i x}(U)=C$ and $F_{i x}(V)=Q$. Assume that $U-I$ and $V-I$ are demiclosed at origin. Let $B: H \rightarrow H$ be $\eta$-strongly monotone and $k$-Lipschitzian on $H$ with $k>0, \eta>0$, and $f$ : $H \rightarrow H$ is a contractive mapping with constant $\beta \in(0,1)$. Assume that $\left\{x_{n}\right\}$ is the sequence given by Algorithm 4 with $\gamma \in(0,1 / \lambda), 0<\varrho_{i}<\omega_{n}<1 / 2$, and $i=1,2$ such that $0<\liminf _{n \rightarrow \infty} \omega_{n} \leq \limsup _{n \rightarrow \infty} \omega_{n}<1 / 2$ and $\alpha_{n} \in(0,1)$ such that $\lim _{n \rightarrow \infty} \alpha_{n}=0$ and $\sum_{n=1}^{\infty} \alpha_{n}=\infty$. If $\Gamma \neq \emptyset$, then the sequence $\left\{x_{n}\right\}$ strongly converges to a split common fixed point $y \in \Gamma$, verifying $y=P_{\Gamma}(I-\mu B+\sigma f)(y)$ which equivalently solves the following variational inequality problem:

$$
y \in \Gamma, \quad\left\langle(\mu B-\sigma f) y, x^{*}-y\right\rangle \geq 0, \quad x^{*} \in \Gamma .
$$

Proof. Let $y$ be the solution of (31). From (9) we obtain that $x_{n+1}-x_{n}+\alpha_{n}\left(\mu B x_{n}-\sigma f\left(x_{n}\right)\right)=\left(I-\mu \alpha_{n} B\right)\left(T_{\omega_{n}} x_{n}-x_{n}\right)$,

hence

$$
\begin{aligned}
\left\langle x_{n+1}-\right. & \left.x_{n}+\alpha_{n}\left(\mu B x_{n}-\sigma f\left(x_{n}\right)\right), x_{n}-y\right\rangle \\
= & \left\langle\left(I-\mu \alpha_{n} B\right)\left(T_{\omega_{n}} x_{n}-x_{n}\right), x_{n}-y\right\rangle \\
= & \left(1-\alpha_{n}\right)\left\langle T_{\omega_{n}} x_{n}-x_{n}, x_{n}-y\right\rangle \\
& +\alpha_{n}\left\langle(I-\mu B)\left(T_{\omega_{n}} x_{n}-x_{n}\right), x_{n}-y\right\rangle \\
\leq & \left(1-\alpha_{n}\right)\left\langle T_{\omega_{n}} x_{n}-x_{n}, x_{n}-y\right\rangle \\
& +\alpha_{n}\left\|(I-\mu B)\left(T_{\omega_{n}} x_{n}-x_{n}\right)\right\|\left\|x_{n}-y\right\| \\
\leq & \left(1-\alpha_{n}\right)\left\langle T_{\omega_{n}} x_{n}-x_{n}, x_{n}-y\right\rangle \\
& +\alpha_{n}(1-\tau)\left\|T_{\omega_{n}} x_{n}-x_{n}\right\|\left\|x_{n}-y\right\| \\
= & \left(1-\alpha_{n}\right)\left\langle T_{\omega_{n}} x_{n}-x_{n}, x_{n}-y\right\rangle \\
& +\omega_{n} \alpha_{n}(1-\tau)\left\|T x_{n}-x_{n}\right\|\left\|x_{n}-y\right\| .
\end{aligned}
$$

By (28), we obtain that

$$
\begin{aligned}
\left\langle x_{n}-T_{\omega_{n}} x_{n}, x_{n}-y\right\rangle= & \frac{\omega_{n}^{2}}{2}\left\|x_{n}-T_{\omega_{n}} x_{n}\right\|^{2} \\
& +\frac{1}{2}\left\|x_{n}-y\right\|^{2}-\frac{1}{2}\left\|T_{\omega_{n}} x_{n}-y\right\|^{2} \\
\geq & \frac{\omega_{n}^{2}}{2}\left\|x_{n}-T x_{n}\right\|^{2}+\frac{1}{2}\left\|x_{n}-y\right\|^{2} \\
& -\frac{1}{2}\left\|x_{n}-y\right\|^{2} \\
& +\frac{\omega_{n}}{2}\left(1-\varrho_{2}-\omega_{n}\right)\left\|x_{n}-T x_{n}\right\|^{2} \\
= & \frac{\omega_{n}\left(1-\varrho_{2}\right)}{2}\left\|x_{n}-T x_{n}\right\|^{2} .
\end{aligned}
$$

It follows from (33) that

$$
\begin{aligned}
\left\langle x_{n+1}-\right. & \left.x_{n}+\alpha_{n}\left(\mu B x_{n}-\sigma f\left(x_{n}\right)\right), x_{n}-y\right\rangle \\
\leq & -\frac{\omega_{n}}{2}\left(1-\varrho_{2}\right)\left(1-\alpha_{n}\right)\left\|x_{n}-T x_{n}\right\|^{2} \\
& +\omega_{n} \alpha_{n}(1-\tau)\left\|T x_{n}-x_{n}\right\|\left\|x_{n}-y\right\|
\end{aligned}
$$

or equivalently

$$
\begin{aligned}
-\left\langle x_{n}-x_{n+1}, x_{n}-y\right\rangle \leq & -\alpha_{n}\left\langle(\mu B-\sigma f) x_{n}, x_{n}-y\right\rangle \\
& -\frac{\omega_{n}}{2}\left(1-\varrho_{2}\right)\left(1-\alpha_{n}\right)\left\|x_{n}-T x_{n}\right\|^{2} \\
& +\omega_{n} \alpha_{n}(1-\tau)\left\|T x_{n}-x_{n}\right\|\left\|x_{n}-y\right\| .
\end{aligned}
$$

Furthermore, using the classical equality (iii) in Lemma 10 and setting $\mathscr{T}_{n}=(1 / 2)\left\|x_{n}-y\right\|^{2}$, we have

$$
\left\langle x_{n}-x_{n+1}, x_{n}-y\right\rangle=\mathscr{T}_{n}-\mathscr{T}_{n+1}+\frac{1}{2}\left\|x_{n}-x_{n+1}\right\|^{2} .
$$

So that (36) can be equivalently rewritten as

$$
\begin{aligned}
\mathscr{T}_{n+1}- & \mathscr{T}_{n}-\frac{1}{2}\left\|x_{n}-x_{n+1}\right\|^{2} \\
\leq & -\alpha_{n}\left\langle(\mu B-\sigma f) x_{n}, x_{n}-y\right\rangle \\
& -\frac{\omega_{n}}{2}\left(1-\varrho_{2}\right)\left(1-\alpha_{n}\right)\left\|x_{n}-T x_{n}\right\|^{2} \\
& +\omega_{n} \alpha_{n}(1-\tau)\left\|T x_{n}-x_{n}\right\|\left\|x_{n}-y\right\| .
\end{aligned}
$$

Now using (32) again, we have

$$
\begin{aligned}
\left\|x_{n+1}-x_{n}\right\|^{2}= & \| \alpha_{n}\left(\sigma f\left(x_{n}\right)-\mu B x_{n}\right) \\
& +\left(I-\mu \alpha_{n} B\right)\left(T_{\omega_{n}} x_{n}-x_{n}\right) \|^{2} .
\end{aligned}
$$

Since $B: H \rightarrow H$ is $\eta$-strongly monotone and $k$-Lipschitzian on $H$, hence it is a classical matter to see that

$$
\begin{aligned}
\left\|x_{n+1}-x_{n}\right\|^{2} \leq & 2 \alpha_{n}^{2}\left\|\sigma f\left(x_{n}\right)-\mu B x_{n}\right\|^{2} \\
& +2\left(1-\alpha_{n} \tau\right)^{2}\left\|T_{\omega_{n}} x_{n}-x_{n}\right\|^{2},
\end{aligned}
$$

which by $\left\|T_{\omega_{n}} x_{n}-x_{n}\right\|=\omega_{n}\left\|x_{n}-T x_{n}\right\|$ yields

$$
\begin{aligned}
\frac{1}{2}\left\|x_{n+1}-x_{n}\right\|^{2} \leq & \alpha_{n}^{2}\left\|\sigma f\left(x_{n}\right)-\mu B x_{n}\right\|^{2} \\
& +\left(1-\alpha_{n} \tau\right)^{2} \omega_{n}^{2}\left\|x_{n}-T x_{n}\right\|^{2} .
\end{aligned}
$$

Then from (38) and (41), we have

$$
\begin{aligned}
\mathscr{T}_{n+1}- & \mathscr{T}_{n}+\left[\frac{\omega_{n}}{2}\left(1-\varrho_{2}\right)\left(1-\alpha_{n}\right)-\omega_{n}^{2}\left(1-\alpha_{n} \tau\right)^{2}\right] \\
\times & \left\|x_{n}-T x_{n}\right\|^{2} \\
\leq & \alpha_{n}\left[\alpha_{n}\left\|\sigma f\left(x_{n}\right)-\mu B x_{n}\right\|^{2}\right. \\
& -\left\langle(\mu B-\sigma f) x_{n}, x_{n}-y\right\rangle \\
& \left.+\omega_{n}(1-\tau)\left\|T x_{n}-x_{n}\right\|\left\|x_{n}-y\right\|\right] .
\end{aligned}
$$

The rest of the proof will be divided into two parts. 
Case 1. Suppose that there exists $n_{0}$ such that $\left\{\mathscr{T}_{n}\right\}_{n \geq n_{0}}$ is nonincreasing. In this situation, $\left\{\mathscr{T}_{n}\right\}$ is then convergent because it is also nonnegative (hence it is bounded from below), so that $\lim _{n \rightarrow \infty}\left(\mathscr{T}_{n+1}-\mathscr{T}_{n}\right)=0$; hence, in light of (42) together with $\lim _{n \rightarrow \infty} \alpha_{n}=0$, the boundedness of $\left\{x_{n}\right\}$ and $0<\liminf _{n \rightarrow \infty} \omega_{n} \leq \lim \sup _{n \rightarrow \infty} \omega_{n}<1 / 2$, we obtain

$$
\lim _{n \rightarrow \infty}\left\|x_{n}-T x_{n}\right\|=0 \text {. }
$$

It also follows from (42) that

$$
\begin{aligned}
\mathscr{T}_{n}-\mathscr{T}_{n+1} \geq \alpha_{n}( & -\alpha_{n}\left\|\sigma f\left(x_{n}\right)-\mu B x_{n}\right\|^{2} \\
& +\left\langle(\mu B-\sigma f) x_{n}, x_{n}-y\right\rangle \\
& \left.-\omega_{n}(1-\tau)\left\|T x_{n}-x_{n}\right\|\left\|x_{n}-y\right\|\right) .
\end{aligned}
$$

Then, by $\sum_{n=0}^{\infty} \alpha_{n}=\infty$, we obviously deduce that

$$
\begin{aligned}
\liminf _{n \rightarrow \infty} \alpha_{n}( & -\alpha_{n}\left\|\sigma f\left(x_{n}\right)-\mu B x_{n}\right\|^{2} \\
& +\left\langle(\mu B-\sigma f) x_{n}, x_{n}-y\right\rangle \\
& \left.-\omega_{n}(1-\tau)\left\|T x_{n}-x_{n}\right\|\left\|x_{n}-y\right\|\right) \leq 0
\end{aligned}
$$

or equivalently (as $\alpha_{n}\left\|\sigma f\left(x_{n}\right)-\mu B x_{n}\right\|^{2} \rightarrow 0$ and $\left.\lim _{n \rightarrow \infty}\left(\mathscr{T}_{n+1}-\mathscr{T}_{n}\right)=0\right)$. From (45), we get

$$
\liminf _{n \rightarrow \infty}\left\langle(\mu B-\sigma f) x_{n}, x_{n}-y\right\rangle \leq 0
$$

Moreover, by Lemma 8, we have

$$
\begin{gathered}
2(\mu \eta-\sigma \beta) \mathscr{T}_{n}+\left\langle(\mu B-\sigma f) y, x_{n}-y\right\rangle \\
\leq\left\langle(\mu B-\sigma f) x_{n}, x_{n}-y\right\rangle
\end{gathered}
$$

which by (46) entails

$$
\liminf _{n \rightarrow \infty}\left\langle 2(\mu \eta-\sigma \beta) \mathscr{T}_{n}+(\mu B-\sigma f) y, x_{n}-y\right\rangle \leq 0
$$

Hence, recalling that $\lim _{n \rightarrow \infty} \mathscr{T}_{n}$ exists, we equivalently obtain

$$
2(\mu \eta-\sigma \beta) \lim _{n \rightarrow \infty} \mathscr{T}_{n}+\liminf _{n \rightarrow \infty}\left\langle(\mu B-\sigma f) y, x_{n}-y\right\rangle \leq 0,
$$

Namely,

$$
2(\mu \eta-\sigma \beta) \lim _{n \rightarrow \infty} \mathscr{T}_{n} \leq-\liminf _{n \rightarrow \infty}\left\langle(\mu B-\sigma f) y, x_{n}-y\right\rangle .
$$

Now we prove that

$$
\liminf _{n \rightarrow \infty}\left\langle(\mu B-\sigma f) y, x_{n}-y\right\rangle \leq 0 .
$$

It follows from (27) and (43) that

$$
\begin{aligned}
& \gamma\left(\left(1-\varrho_{2}\right)-\lambda \gamma\right)\left\|(V-I) A x_{n}\right\|^{2} \\
& \leq\left\|x_{n}-y\right\|^{2}-\left\|T x_{n}-y\right\|^{2} \\
&=\left(\left\|x_{n}-y\right\|-\left\|T x_{n}-y\right\|\right) \\
& \times\left(\left\|x_{n}-y\right\|+\left\|T x_{n}-y\right\|\right) \\
&=\left(\left\|x_{n}-T x_{n}\right\|\right)\left(\left\|x_{n}-y\right\|+\left\|T x_{n}-y\right\|\right) \\
& \longrightarrow 0, \quad(n \rightarrow \infty),
\end{aligned}
$$

and hence

$$
\lim _{n \rightarrow \infty}\left\|(V-I) A x_{n}\right\|=0 .
$$

Taking $x^{*} \in \omega_{w}\left(x_{n}\right)$, from the demiclosedness of $V-I$ at 0 , we have

$$
V\left(A x^{*}\right)=A x^{*}
$$

Now, by setting $u_{n}=x_{n}+\gamma A^{*} V-I A x_{n}$, it follows that $x^{*} \epsilon$ $\omega_{w}\left(u_{n}\right)$. On the other hand,

$$
\begin{aligned}
\left\|U\left(u_{n}\right)-u_{n}\right\| & =\left\|T x_{n}-x_{n}-\gamma A^{*} V-I A x_{n}\right\| \\
& \leq\left\|T x_{n}-x_{n}\right\|+\gamma\left\|A^{*}\right\|\left\|V-I A x_{n}\right\| \rightarrow 0,
\end{aligned}
$$

which, combined with the demiclosedness of $U-I$ at 0 , yields

$$
U x^{*}=x^{*} \text {. }
$$

Hence, $x^{*} \in C$ and $x^{*} \in \Gamma$. We can take subsequence $\left\{x_{n_{j}}\right\}$ of $\left\{x_{n}\right\}$ such that $\lim _{n \rightarrow \infty} x_{n_{j}}=x^{*}$ and

$$
\liminf _{n \rightarrow \infty}\left\langle(\sigma f-\mu B) y, x_{n}-y\right\rangle=\lim _{j \rightarrow \infty}\left\langle(\sigma f-\mu B) y, x_{n_{j}}-y\right\rangle,
$$

which leads to

$$
\liminf _{n \rightarrow \infty}\left\langle(\sigma f-\mu B) y, x_{n}-y\right\rangle=\left\langle(\sigma f-\mu B) y, x^{*}-y\right\rangle \leq 0 .
$$

By (50), we have $\lim _{n \rightarrow \infty} \Gamma_{n}=0$, and hence $\left\{x_{n}\right\}$ converges strongly to $y$.

Case 2. Suppose that there exists a subsequence $\left\{\mathscr{T}_{n_{k}}\right\}_{k \geq 0}$ of $\left\{\mathscr{T}_{n}\right\}_{n \geq 0}$ such that $\mathscr{T}_{n_{k}} \leq \mathscr{T}_{n_{k+1}}$ for all $k \geq 0$. In this situation, we consider the sequence of indices $\{\delta(n)\}$ as defined in Lemma 11. It follows that $\mathscr{T}_{\delta(n+1)}-\mathscr{T}_{\delta(n)}>0$, which by $(42)$ amounts to

$$
\begin{gathered}
{\left[\frac{\omega_{n}}{2}\left(1-\varrho_{2}\right)\left(1-\alpha_{\delta(n)}\right)-\omega_{n}^{2}\left(1-\alpha_{\delta(n)} \tau\right)^{2}\right]\left\|x_{\delta(n)}-T x_{\delta(n)}\right\|^{2}} \\
\leq \alpha_{\delta(n)}\left[\alpha_{\delta(n)}\left\|\sigma f\left(x_{\delta(n)}\right)-\mu B x_{n}\right\|^{2}\right. \\
-\left\langle(\mu B-\sigma f) x_{n}, x_{n}-y\right\rangle \\
\left.+\omega_{n}(1-\tau)\left\|T x_{\delta(n)}-x_{\delta(n)}\right\|\left\|x_{\delta(n)}-y\right\|\right]
\end{gathered}
$$


By the boundedness of $\left\{x_{n}\right\}$ and $\lim _{n \rightarrow \infty} \alpha_{n}=0$, we immediately obtain

$$
\lim _{n \rightarrow \infty}\left\|x_{\delta(n)}-T x_{\delta(n)}\right\|=0
$$

Using (9), we have

$$
\begin{aligned}
\left\|x_{\delta(n)+1}-x_{\delta(n)}\right\| \leq & \alpha_{\delta(n)}\left\|\sigma f\left(x_{\delta(n)}\right)-\mu B x_{\delta(n)}\right\| \\
& +\left|1-\alpha_{\delta(n)} \tau\right|\left\|T_{\omega_{n}} x_{\delta(n)}-x_{\delta(n)}\right\| \\
\leq & \alpha_{\delta(n)}\left\|\sigma f\left(x_{\delta(n)}\right)-\mu B x_{\delta(n)}\right\| \\
& +\left|1-\alpha_{\delta(n)} \omega_{n} \tau\right|\left\|T x_{\delta(n)}-x_{\delta(n)}\right\|,
\end{aligned}
$$

which together with $(60)$ and $\lim _{n \rightarrow \infty} \alpha_{n}=0$ yields

$$
\lim _{n \rightarrow \infty}\left\|x_{\delta(n)+1}-x_{\delta(n)}\right\|=0 .
$$

Similar to Case 1, we have

$$
\liminf _{n \rightarrow \infty}\left\langle(\mu B-\sigma f) y, x_{\delta(n)}-y\right\rangle \geq 0 .
$$

Now by (59) we clearly have

$$
\begin{gathered}
\alpha_{\delta(n)}\left\|\sigma f\left(x_{\delta(n)}\right)-\mu B x_{\delta(n)}\right\|^{2}+\alpha(1-\tau) \\
\times\left\|T x_{\delta(n)}-x_{\delta(n)}\right\|\left\|x_{\delta(n)}-y\right\| \\
\geq\left\langle(\mu B-\sigma f) x_{\delta(n)}, x_{\delta(n)}-y\right\rangle
\end{gathered}
$$

which in the light of (47) yields

$$
\begin{aligned}
2(\mu \eta-\sigma \beta) \mathscr{T}_{\delta(n)}+\left\langle(\mu B-\sigma f) y, x_{\delta(n)}-y\right\rangle \\
\leq \alpha_{\delta(n)}\left\|\sigma f\left(x_{\delta(n)}\right)-\mu B x_{\delta(n)}\right\|^{2} \\
\quad+\alpha(1-\tau)\left\|T x_{\delta(n)}-x_{\delta(n)}\right\|\left\|x_{\delta(n)}-y\right\| .
\end{aligned}
$$

Hence (as $\lim _{n \rightarrow \infty} \alpha_{\delta(n)}\left\|\sigma f\left(x_{\delta(n)}\right)-\mu B x_{\delta(n)}\right\|^{2}=0$ and $\left.\lim _{n \rightarrow \infty}\left\|T x_{\delta(n)}-x_{\delta(n)}\right\|=0\right)$ it follows that

$$
2(\mu \eta-\sigma \beta) \limsup _{n \rightarrow \infty} \mathscr{T}_{\delta(n)} \leq-\liminf _{n \rightarrow \infty}\left\langle(\mu B-\sigma f) y, x_{\delta(n)}-y\right\rangle .
$$

From (59) and (63), we obtain

$$
\lim _{n \rightarrow \infty}\left\langle(\mu B-\sigma f) y, x_{\delta(n)}-y\right\rangle \geq 0,
$$

which by (60) yields $\lim \sup _{n \rightarrow \infty} \mathscr{T}_{\delta(n)}=0$, so that $\lim _{n \rightarrow \infty} \mathscr{T}_{\delta(n)}=0$. Combining $(62)$, we have $\lim _{n \rightarrow \infty} \mathscr{T}_{\delta(n)+1}$ $=0$. Then, recalling that $\mathscr{T}_{n}<\mathscr{T}_{\delta(n)+1}$ (by Lemma 11), we get $\lim _{n \rightarrow \infty} \mathscr{T}_{n}=0$, so that $x_{n} \rightarrow y$ strongly.

In addition, the variational inequality (50) and (67) can be written as

$$
\left\langle(I-\mu B+\sigma f) y-y, x^{*}-y\right\rangle \leq 0, \quad x^{*} \in \Gamma .
$$

So, by the Lemma 12, it is equivalent to the fixed point equation

$$
P_{\Gamma}(I-\mu B+\sigma f) y=y .
$$

\section{Application in Other Nonlinear Operators}

In order to define our motivations, we recall some definitions of classed of operators as follows

Definition 15. $T: D(T) \subseteq H \rightarrow H$ is said to be

(1) nonspreading in $[26,27]$, if

$$
\|T x-T y\|^{2} \leq\|T x-y\|^{2}+\|T y-x\|^{2}, \quad \forall x, y \in C,
$$

(2) demicontractive in [28], if there exists a constant $\alpha<$ 1 such that

$$
\begin{array}{r}
\|T x-q\|^{2} \leq\|x-q\|^{2}+\alpha\|x-T x\|^{2}, \\
\forall(x, q) \in H \times F_{i x}(T) .
\end{array}
$$

Remark 16. Iemoto and Takahashi [29] proved that (70) is equivalent to

$$
\begin{array}{r}
\|T x-T y\|^{2} \leq\|x-y\|^{2}+2\langle x-T x, y-T y\rangle, \\
\forall x, y \in C .
\end{array}
$$

Iterative methods for nonspreading mapping have been extensively investigated; see [30-34].

Remark 17. From the Definition 5 (3), Definition 7, and Definition 15, we have the following facts.

(i) Observe that every nonspreading mapping is 0strictly pseudononspreading.

(ii) If $T$ is nonspreading mapping and the set of fixed point is nonempty, then $T$ is quasi-nonexpansive mapping.

(iii) Every pseudononspreading mapping with a nonempty fixed point set $F_{i x}(T)$ is demicontractive (see [28]).

Corollary 18. Given a bounded linear operator $A: H_{1} \rightarrow$ $H_{2}$, let $U: H_{1} \rightarrow H_{1}$ and $V: H_{2} \rightarrow H_{2}$ be two nonspreading mappings with fixed point $F_{i x}(U)=C$ and $F_{i x}(V)=Q$. Assume that $U-I$ and $V-I$ are demiclosed at origin. Let $B: H \rightarrow H$ be $\eta$-strongly monotone and $k$-Lipschitzian on $H$ with $k>0$, $\eta>0$, and let $f: H \rightarrow H$ be a contractive mapping with constant $\beta \in(0,1)$. Let $\left\{x_{n}\right\}$ be the sequence given by (9) with $\gamma \in(0,1 / \lambda), 0<\varrho_{i}<\omega_{n}<1 / 2$, and $i=1,2$ such that $0<\liminf _{n \rightarrow \infty} \omega_{n} \leq \limsup _{n \rightarrow \infty} \omega_{n}<1 / 2$ and $\alpha_{n} \in(0,1)$ such that $\lim _{n \rightarrow \infty} \alpha_{n}=0$ and $\sum_{n=1}^{\infty} \alpha_{n}=\infty$. If $\Gamma \neq \emptyset$, then the sequence $\left\{x_{n}\right\}$ strongly converges to a split common fixed point $x^{*} \in \Gamma$, verifying $x^{*}=P_{\Gamma}(I-\mu B+\sigma f)\left(x^{*}\right)$ which equivalently solves the following variational inequality problem:

$$
x^{*} \in \Gamma, \quad\left\langle(\mu B-\sigma f) x^{*}, y-x^{*}\right\rangle \geq 0, \quad y \in \Gamma .
$$

Proof. Form the proof of the Theorem 14, we can easily certify this theorem by nonspreading mapping (i.e., nonspreading is 0 -strictly pseudononspreading).

From the Remark 17(ii) and the Corollary 18, we have the following corollary. 
Corollary 19. Given a bounded linear operator $A: H_{1} \rightarrow$ $H_{2}$, let $U: H_{1} \rightarrow H_{1}$ and $V: H_{2} \rightarrow H_{2}$ be two quasinonexpansives with fixed point $F_{i x}(U)=C$ and $F_{i x}(V)=Q$. Assume that $U-I$ and $V-I$ are demiclosed at origin. Let $B: H \rightarrow H$ be $\eta$-strongly monotone and $k$-Lipschitzian on $H$ with $k>0, \eta>0$, and let $f: H \rightarrow H$ be a contractive mapping with constant $\beta \in(0,1)$. Let $\left\{x_{n}\right\}$ be the sequence given by (9) with $\gamma \in(0,1 / \lambda), \omega_{n} \in(0,1 / 2)$ such that $0<$ $\liminf _{n \rightarrow \infty} \omega_{n} \leq \limsup _{n \rightarrow \infty} \omega_{n}<1 / 2$ and $\alpha_{n} \in(0,1)$ such that $\lim _{n \rightarrow \infty} \alpha_{n}=0$ and $\sum_{n=1}^{\infty} \alpha_{n}=\infty$. If $\Gamma \neq \emptyset$, then the sequence $\left\{x_{n}\right\}$ strongly converges to a split common fixed point $x^{*} \in \Gamma$, verifying $x^{*}=P_{\Gamma} f\left(x^{*}\right)$ which equivalently solves the following variational inequality problem:

$$
x^{*} \in \Gamma, \quad\left\langle(\mu B-\sigma f) x^{*}, y-x^{*}\right\rangle \geq 0, \quad y \in \Gamma .
$$

If $\sigma=\mu=1$ and $B=I$ in (9), thus $k=\eta=1$ and $\beta \epsilon$ $(0,1 / 2)$, and then we obtain (8) and the following corollary. On the other hand, this corollary was proven by Zhao and $\mathrm{He}$ [14].

Corollary 20. Given a bounded linear operator $A: H_{1} \rightarrow$ $H_{2}$, let $U: H_{1} \rightarrow H_{1}$ and $V: H_{2} \rightarrow H_{2}$ be two quasinonexpansives with fixed point $F_{i x}(U)=C$ and $F_{i x}(V)=Q$. Assume that $U-I$ and $V-I$ are demiclosed at origin. Let $f$ : $H \rightarrow H$ be a contractive mapping with constant $\beta \in(0,1 / 2)$. Let $\left\{x_{n}\right\}$ be the sequence given by (8) with $\gamma \in(0,1 / \lambda), \omega_{n} \epsilon$ $(0,1 / 2)$ such that $0<\liminf _{n \rightarrow \infty} \omega_{n} \leq \limsup _{n \rightarrow \infty} \omega_{n}<$ $1 / 2$, and $\alpha_{n} \in(0,1)$ such that $\lim _{n \rightarrow \infty} \alpha_{n}=0$ and $\sum_{n=1}^{\infty} \alpha_{n}=$ $\infty$. If $\Gamma \neq \emptyset$, then the sequence $\left\{x_{n}\right\}$ strongly converges to a split common fixed point $x^{*} \in \Gamma$, verifying $x^{*}=P_{\Gamma}(I-\mu B+\sigma f)\left(x^{*}\right)$ which equivalently solves the following variational inequality problem:

$$
x^{*} \in \Gamma, \quad\left\langle(I-f) x^{*}, y-x^{*}\right\rangle \geq 0, \quad y \in \Gamma .
$$

\section{References}

[1] Y. Censor and T. Elfving, "A multiprojection algorithm using Bregman projections in a product space," Numerical Algorithms, vol. 8, no. 2-4, pp. 221-239, 1994.

[2] C. Byrne, "Iterative oblique projection onto convex sets and the split feasibility problem," Inverse Problems, vol. 18, no. 2, pp. 441453, 2002.

[3] Y. Censor, T. Elfving, N. Kopf, and T. Bortfeld, “The multiplesets split feasibility problem and its applications for inverse problems," Inverse Problems, vol. 21, no. 6, pp. 2071-2084, 2005.

[4] Y. Censor, T. Bortfeld, B. Martin, and A. Trofimov, "Aunified approach for inversion problems in intensity modulated radiation therapy," Physics in Medicine and Biology, vol. 51, no. 10, pp. 2353-2365, 2006.

[5] Y. Censor, A. Motova, and A. Segal, "Perturbed projections and subgradient projections for the multiple-sets split feasibility problem," Journal of Mathematical Analysis and Applications, vol. 327, no. 2, pp. 1244-1256, 2007.

[6] G. Lopez, V. Martin, and H. K. Xu, "Iterative algorithms for the multiple-sets split feasibility problem," in Biomedical Mathematics: Promising Directions in Imaging, Therapy Planning and Inverse Problems, Y. Censor, M. Jiang, and G. Wang, Eds., pp. 243-279, Medical Physics Publishing, Madison, Wis, USA, 2009.
[7] F. Wang and H.-K. Xu, "Cyclic algorithms for split feasibility problems in Hilbert spaces," Nonlinear Analysis: Theory, Methods \& Applications, vol. 74, no. 12, pp. 4105-4111, 2011.

[8] H.-K. Xu, "A variable Krasnoselskii-Mann algorithm and the multiple-set split feasibility problem," Inverse Problems, vol. 22, no. 6, pp. 2021-2034, 2006.

[9] L. Landweber, "An iteration formula for Fredholm integral equations of the first kind," American Journal of Mathematics, vol. 73, pp. 615-624, 1951.

[10] H.-K. Xu, "Iterative methods for the split feasibility problem in infinite-dimensional Hilbert spaces," Inverse Problems, vol. 26, no. 10, Article ID 105018, 17 pages, 2010.

[11] A. Moudafi, "The split common fixed-point problem for demicontractive mappings," Inverse Problems, vol. 26, no. 5, Article ID 055007, 6 pages, 2010.

[12] Y. Censor and A. Segal, "The split common fixed point problem for directed operators," Journal of Convex Analysis, vol. 16, no. 2, pp. 587-600, 2009.

[13] A. Moudafi, "A note on the split common fixed-point problem for quasi-nonexpansive operators," Nonlinear Analysis: Theory, Methods \& Applications, vol. 74, no. 12, pp. 4083-4087, 2011.

[14] J. Zhao and S. He, "Strong convergence of the viscosity approximation process for the split common fixed-point problem of quasi-nonexpansive mappings," Journal of Applied Mathematics, vol. 2012, Article ID 438023, 12 pages, 2012.

[15] B.-C. Deng, T. Chen, and B. Xin, "Parallel and cyclic algorithms for quasi-nonexpansives in Hilbert space," Abstract and Applied Analysis, vol. 2012, Article ID 218341, 27 pages, 2012.

[16] P.-E. Maingé, “The viscosity approximation process for quasinonexpansive mappings in Hilbert spaces," Computers \& Mathematics with Applications, vol. 59, no. 1, pp. 74-79, 2010.

[17] I. Yamada and N. Ogura, "Hybrid steepest descent method for variational inequality problem over the fixed point set of certain quasi-nonexpansive mappings," Numerical Functional Analysis and Optimization, vol. 25, no. 7-8, pp. 619-655, 2004.

[18] F. E. Browder and W. V. Petryshyn, "Construction of fixed points of nonlinear mappings in Hilbert space," Journal of Mathematical Analysis and Applications, vol. 20, pp. 197-228, 1967.

[19] K. Aoyama and F. Kohsaka, "Fixed point and mean convergence theorems for a family of $\lambda$-hybrid mappings," Journal of Nonlinear Analysis and Optimization. Theory and Applications, vol. 2, no. 1, pp. 87-95, 2011.

[20] K. Aoyama, "Halpern's iteration for a sequence of quasinonexpansive type mappings," Non-Linear Mathematics for Uncertainty and Its Applications, vol. 100, pp. 387-394, 2011.

[21] B.-C. Deng, T. Chen, and Z.-F. Li, "Cyclic iterative method for strictly pseudononspreading in Hilbert space," Journal of Applied Mathematics, vol. 2012, Article ID 435676, 15 pages, 2012.

[22] M. O. Osilike and F. O. Isiogugu, "Weak and strong convergence theorems for nonspreading-type mappings in Hilbert spaces," Nonlinear Analysis: Theory, Methods \& Applications, vol. 74, no. 5, pp. 1814-1822, 2011.

[23] N. Petrot and R. Wangkeeree, "A general iterative scheme for strict pseudononspreading mapping related to optimization problem in Hilbert spaces," Journal of Nonlinear Analysis and Optimization, vol. 2, no. 2, pp. 329-336, 2011.

[24] M. Tian, "A general iterative algorithm for nonexpansive mappings in Hilbert spaces," Nonlinear Analysis: Theory, Methods \& Applications, vol. 73, no. 3, pp. 689-694, 2010. 
[25] P.-E. Maingé, "Strong convergence of projected subgradient methods for nonsmooth and nonstrictly convex minimization," Set-Valued Analysis, vol. 16, no. 7-8, pp. 899-912, 2008.

[26] F. Kohsaka and W. Takahashi, "Fixed point theorems for a class of nonlinear mappings related to maximal monotone operators in Banach spaces," Archiv der Mathematik, vol. 91, no. 2, pp. 166177, 2008.

[27] F. Kohsaka and W. Takahashi, "Existence and approximation of fixed points of firmly nonexpansive-type mappings in Banach spaces," SIAM Journal on Optimization, vol. 19, no. 2, pp. 824$835,2008$.

[28] F. Wang and H.-K. Xu, "Cyclic algorithms for split feasibility problems in Hilbert spaces," Nonlinear Analysis: Theory, Methods \& Applications, vol. 74, no. 12, pp. 4105-4111, 2011.

[29] S. Iemoto and W. Takahashi, "Approximating common fixed points of nonexpansive mappings and nonspreading mappings in a Hilbert space," Nonlinear Analysis: Theory, Methods \& Applications, vol. 71, no. 12, pp. e2082-e2089, 2009.

[30] M. Eslamian, "Convergence theorems for nonspreading mappings and nonexpansive multivalued mappings and equilibrium problems," Optimization Letters, vol. 7, no. 3, pp. 547-557, 2013.

[31] Y. Kurokawa and W. Takahashi, "Weak and strong convergence theorems for nonspreading mappings in Hilbert spaces," Nonlinear Analysis: Theory, Methods \& Applications, vol. 73, no. 6, pp. 1562-1568, 2010.

[32] K. S. Kim, "Approximating common fixed points of nonspreading-type mappings and nonexpansive mappings in a Hilbert space," Abstract and Applied Analysis, vol. 2012, Article ID 594218, 18 pages, 2012.

[33] S. Plubtieng and S. Chornphrom, "Strong convergence theorem for equilibrium problems and fixed points of a nonspreading mapping in Hilbert spaces," Fixed Point Theory and Applications, vol. 2010, Article ID 296759, 16 pages, 2010.

[34] W. Takahashi, N.-C. Wong, and J.-C. Yao, "Fixed point theorems and convergence theorems for generalized nonspreading mappings in Banach spaces," Journal of Fixed Point Theory and Applications, vol. 11, no. 1, pp. 159-183, 2012. 


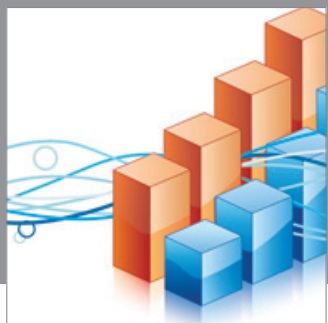

Advances in

Operations Research

mansans

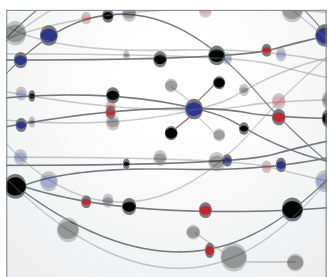

The Scientific World Journal
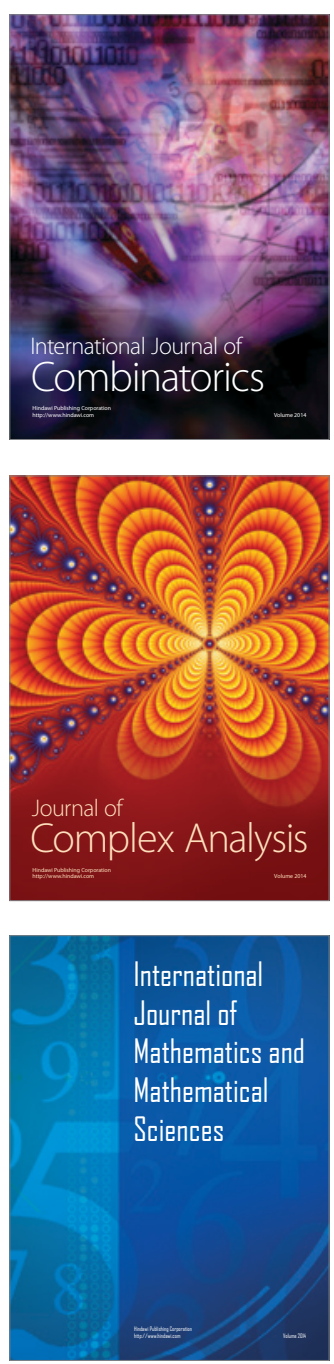
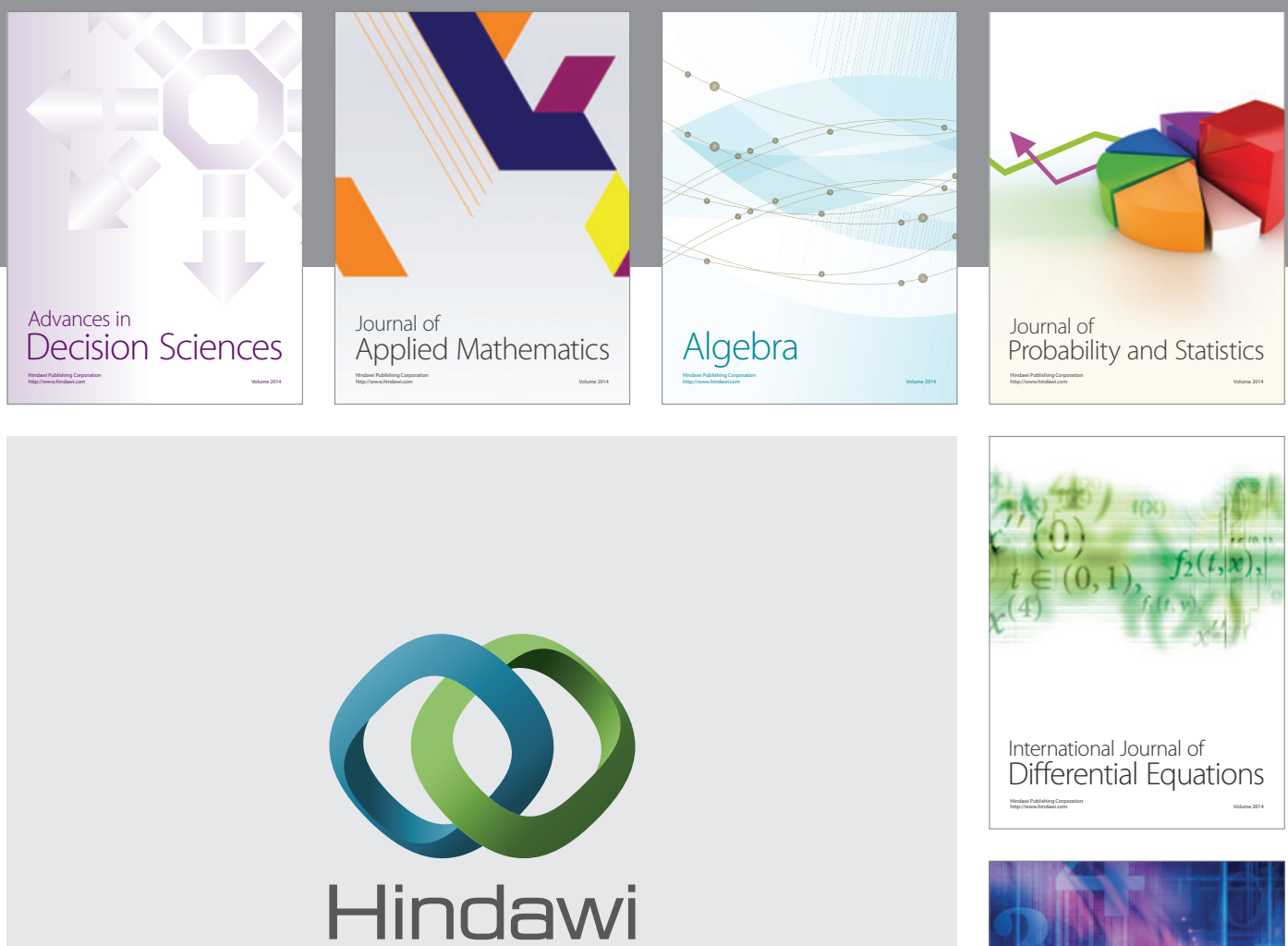

Submit your manuscripts at http://www.hindawi.com
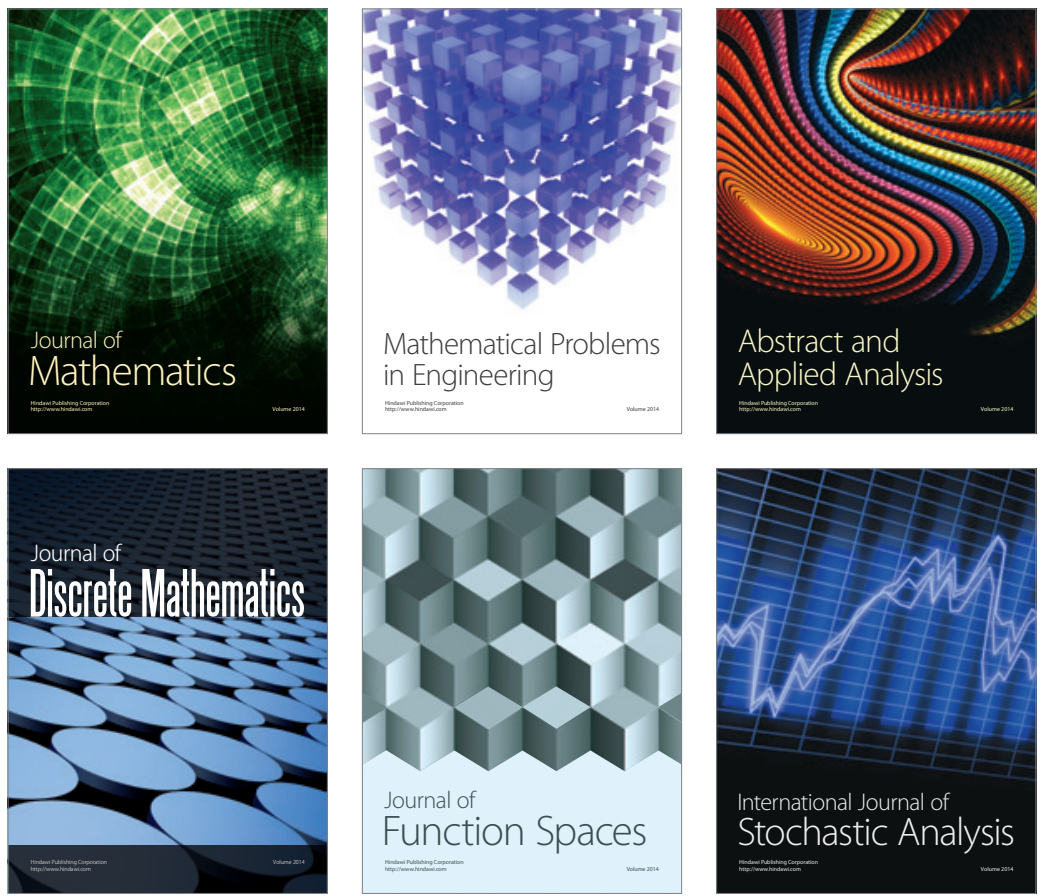

Journal of

Function Spaces

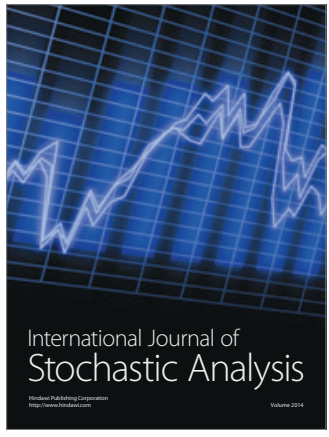

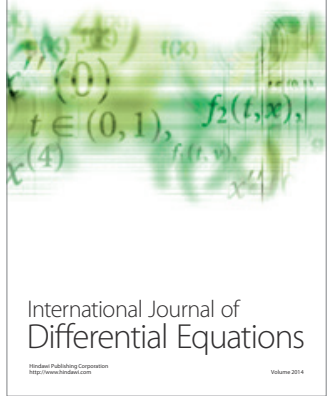
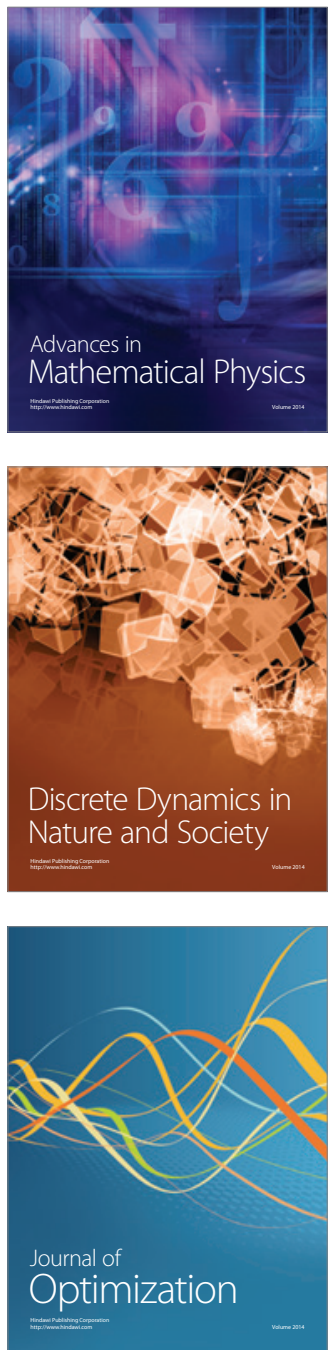